\title{
Design of Logistics Route Planning for Printing Enterprises Based on Baidu Map
}

\author{
Zhong-Yue DA, a , Wen-Jie YANG ${ }^{1}$,Pei-Pei RAN ${ }^{1}$,Xing-Gen QIAN $^{1}$, Shuai SHAO ${ }^{1}$, Shun PAN ${ }^{1}$, \\ Bing-Jie SHI ${ }^{1}$, Yan $\mathrm{LI}^{1}$, Rui-Li HE ${ }^{1}$ and Yong-Hao XIAO ${ }^{1}$ \\ ${ }^{1}$ School of printing and packaging engineering, Beijing Institute of Graphic Communication, Beijing, China
}

\begin{abstract}
As traditional printing industry is facing fierce competition in the market and logistics becomes a new sources of growth, the traditional printing enterprises want to build and improve their logistics system. So in this paper, the author has designed the logistics planning system based on the Baidu map development kit. It has the function of route planning and location tracking. This system meet the printing enterprise logistics route planning and tracking demand.
\end{abstract}

\section{Background}

\subsection{Industry background}

The production of small and medium-sized printing enterprises is based on orders, so they are in a passive position in supply and demand [1]. Most enterprises are faced with high delivery requirements. Therefore, from the perspective of the enterprise logistics, it is the key of the survival and development of enterprises to realize the control of logistics cost and the more efficient management of logistics. Small and medium-sized printing enterprises need to use advanced management ideas and tools to help reduce costs, improve market response speed. In this paper, our aim is to design a route planning software for printing enterprise logistics.

\subsection{Android platform background}

Android occupies the largest mobile terminal operating system market. The open source and free features make it become the priority of the operating system for major mobile phone manufacturers [2]. Besides, it is easy for developers to operate and develop.

\subsection{Baidu Map SDK}

Baidu maps SDK is an application program interface. Developers can call the SDK interface, access to Baidu maps services and build a feature rich, interactive system [3]. The Baidu maps version we use in this paper is V4.0.0, which is based on Android.

\footnotetext{
${ }^{a}$ Corresponding author: dazhongyue@qq.com
} 


\section{System design}

\subsection{Technology platform}

Owing to the advantages of Android, we choose Android as mobile terminal system. The tools that we need are Android studio and MyEclipse, first of which is a good use of Android development tools and last of which is a server development platform. Test environment is the Android device and Window system of the computer. The development tools are shown in Table 1.

Table 1. Development environment

\begin{tabular}{|l|l|}
\hline Demand name & Platform and tools \\
\hline Hardware platform & Android equipment + Computer \\
\hline Development tool & MyEclipse + Android Studio \\
\hline Development language & Java \\
\hline Compile environment & Win7+JDK \\
\hline Testing environment & Android \\
\hline Database & MySQL \\
\hline Software development kit & Baidu Map Android SDK \\
\hline
\end{tabular}

\subsection{Requirement analysis}

In order to find new revenue growth opportunities, the management of printing enterprises hope to improve profits through information management technology. However, their logistics is independent of the information management system. So printing enterprise manager want to have a logistics software which is able to compatible with the information management system. Specific requirements are as follows.

1. This software has route query based on mobile terminal.

2. Company management can know the location location at any time.

3. The path to the client should be recorded in the form of the start and end location.

4. The software should be extensible and compatible with other systems.

\subsection{Design}

\subsubsection{Framework design}

As Figure 1 shown, the whole system is composed of three parts, including Baidu Map Service Center, Mobile terminal and company server. The first part is provided by Baidu Maps API. In the following, we mainly discuss the latter two parts.

Baidu maps service provides route information. Mobile terminal is mainly composed of smart phones and mobile software. It provides man-machine interface and route planning function, while the server is mainly to complete the mobile location tracking and information recording function. Mobile terminal is the most important part of the whole system. So this paper focuses on the development of Android terminal.

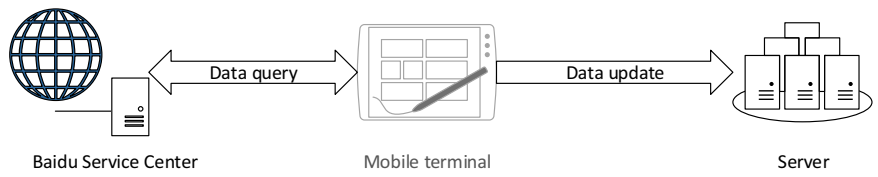

Figure. 1 System framework 


\subsubsection{Mobile terminal design}

From the functional analysis, the client is divided into user login, route query, location coordinates query and upload the current information. The route query and coordinate location query are directly related to the efficiency of the client's distribution. So these functions are completed in the mobile terminal.

According to functional analysis, we divide the mobile terminal into three layers, which are business logic layer, presentation layer and data layer. We not only need to implement the interactive interface, but also to achieve business inquiries and monitoring.

The overall flow chart is shown in Figure two. After landing their account, the user can begin to search route and select the delivery route. In addition, according to needs, the user can choose the location model.

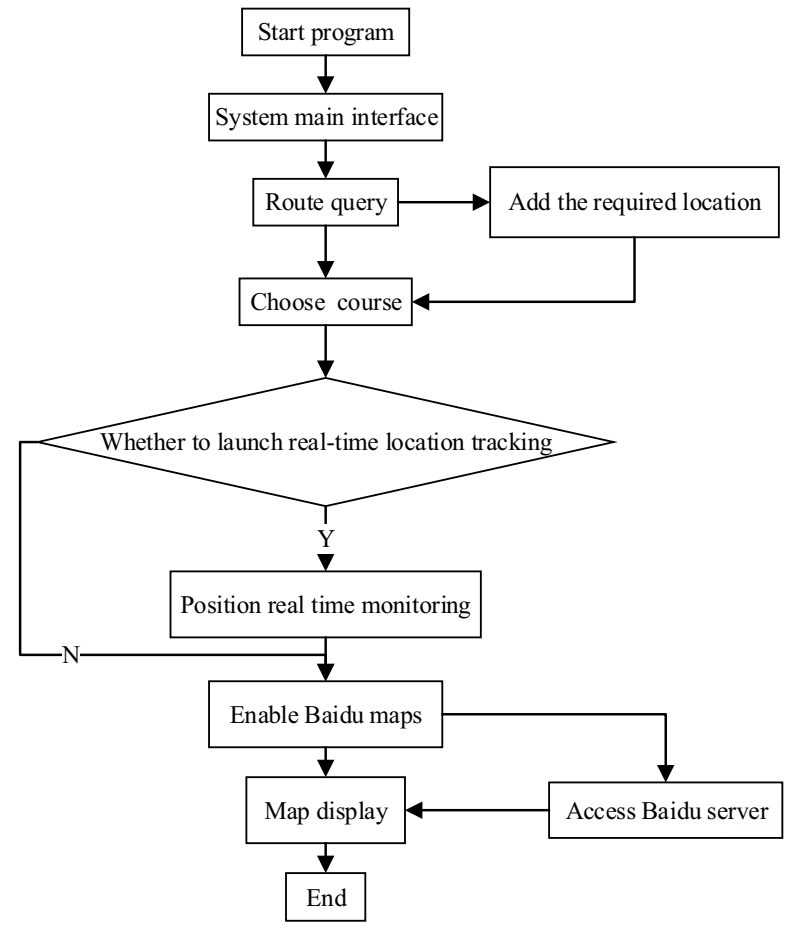

Figure. 2 Overall client flow chart

\subsubsection{Server Design}

Database is the most important part of server. Therefore design of database structure should be completed before server-side programming. The database is not only the support of the system, but also the support of management. The design of database model is the design server model.

According to the demand analysis, this paper designed user, route line two table. Table User is a table to record user information, including account number, password. Table route includes the starting location, the end location, and the distance of the route. 
Table 2. Table Route

\begin{tabular}{|l|l|l|l|}
\hline Route properties & Data type & Meaning & Data sources \\
\hline User & VARCHAR(128) & User name & User ID \\
\hline Start & VARCHAR(128) & Starting position & Mobile terminal input \\
\hline End & VARCHAR(128) & Destination & Mobile terminal input \\
\hline Distance & INT(11) & Route length & Baidu map server \\
\hline Flag & VARCHAR(128) & Logistics type & Mobile terminal \\
\hline Datetime & DATATIME & Recording time & System \\
\hline
\end{tabular}

\section{System implementation}

\subsection{Client implementation}

The design of mobile terminal presentation layer is the interface design of software. The interface of the mobile terminal is mainly composed of the controls provided by Android and the map controls provided by Baidu Map SDK. We use dialog boxes and text boxes and other controls to ensure the simplification of the operation. Business logic layer is activity on Android platform. They implement logic operations and call Baidu maps API and data operation.

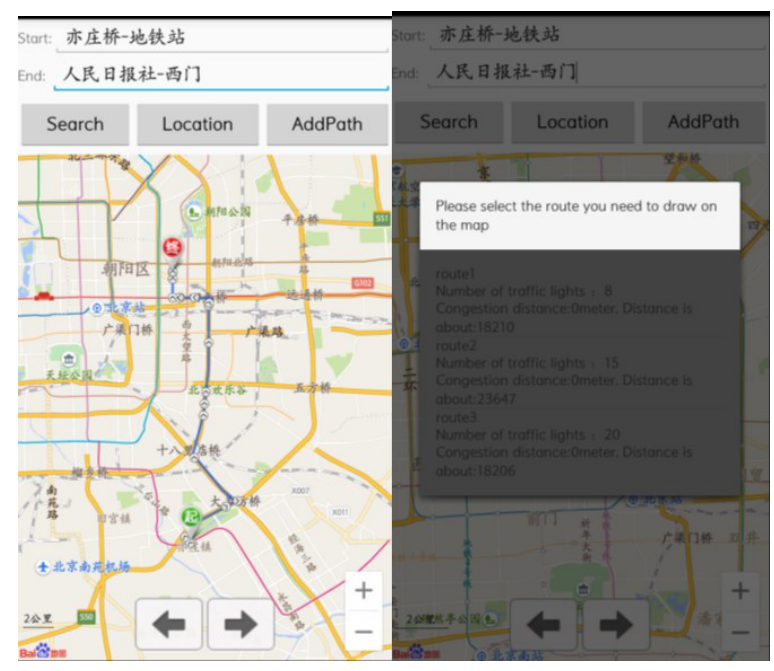

Figure 3. Route plan (left) and Results of the route (right)

The core meaning of the mobile terminal is to complete the route guidance. So we add the location function in the mobile terminal. As shown in Figure 3 and figure 4, the client can complete the basic route query, selection and navigation functions. On the route selection interface, it show the length of the route and the number of traffic lights. It help users choose a better route. In addition, we also add keyword recommendation function, which improve the accuracy of the input address. 


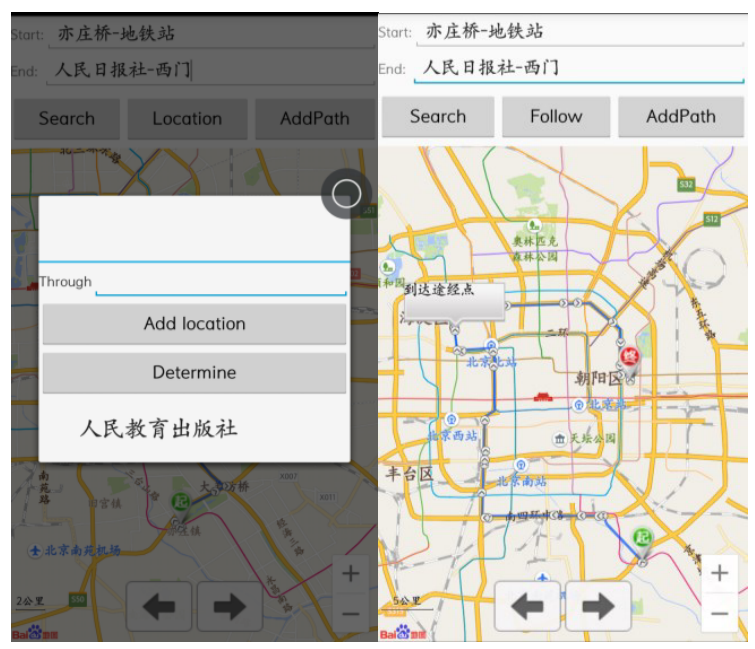

Figure 4. Add locations (left) and results (right)

\subsection{Server side implementation}

The server is designed as two layers, which are business logic layer and data access layer. So, server is mainly data storage and data operation. When the mobile terminal is enabled, the server get the information of the mobile terminal in real time, including the location information and route selection information.

The administrator can understand the situation from the server in real time, and the design and implementation of the server can be merged into the information management system.

\section{Conclusion}

In this paper, we achieve the logistics planning and real-time location tracking by using the Baidu map SDK technology and the corresponding database technology. Mobile terminal map technology improves improve the timeliness and accuracy of logistics distribution. This design has certain significance for printing enterprises to excavate "the third profit source". However, the server administrator can not directly catch sight of the client position. Next, we will improve the server side and establish the relationship between logistics and inventory.

\section{Acknowledgments}

The study is supported by the 'Development and application of micron level vision quality detector (2013 YQ140517)'.

\section{References}

1. $\mathrm{ZH}$ Tao, The design and application of Logistics Oriented Management Information System for small and medium sized enterprises of printing, University of Electronic Science and Technology (2008)

2. ZY Da, Design of Query Software for Printing Enterprise Based On Android Platform (2016)

3. ML Shi, Based on Baidu maps Android application to build. Technology Research and Development, 34-38 (2014) 
4. SL Liu, SZ printing company in the application of ERP demand analysis and process optimization, Shandong University (2012)

5. Baidu maps SDK V 4.0.0[EB/OL]. http://lbsyun.baidu.com/index.php?title=androidsdk.

6. GG Sun, Analysis and design of city logistics distribution system based on GIS. North China Electric Power University (2013)

7. YP Chen, Promotion of the Value Creating Capacity of Agricultural Products Logistics Industry from the Perspective of Resource Development. Journal of Contemporary Finance and Economics, 99-112(2015)

8. Kenneth C. Laudon, Jane P. Laudon, Management Information Systems (13th Edition, 2016)

9. XY Shu, Design of mobile web map service based on Android platform, DMU (2009)

10. ZY Xia, Logistics management of printing enterprises, Print Today(2005: 65-66) 